\title{
Radiative hydrodynamics models of stellar convection
}

\author{
Matthias Steffen ${ }^{1}$ \\ ${ }^{1}$ Astrophysikalisches Institut Potsdam, 14482 Potsdam, Germany \\ email: msteffen@aip.de
}

\begin{abstract}
In the quest for a better theoretical understanding of the dynamical processes operating in stellar envelopes, increasingly more realistic hydrodynamical models of stellar convection have been developed over the last 25 years. Based on 3D state-of-the-art simulations performed recently with the radiation hydrodynamics code $\mathrm{CO}^{5} \mathrm{BOLD}$, we review the present status of hydrodynamical modeling of stellar surface convection and demonstrate the basic differences between 3D hydrodynamical models and 1D 'classical' hydrostatic atmospheres where convection is treated by the mixing-length theory. We briefly discuss some of the many possible applications across the Hertzsprung-Russell diagram, including spectroscopic abundance determinations using 3D stellar atmospheres, particularly with regard to the much debated question of the solar oxygen abundance.
\end{abstract}

Keywords. Convection, hydrodynamics, radiative transfer, Sun: granulation, Sun: abundances, stars: atmospheres

\section{Introduction}

Convection is a universal feature: essentially all types of stars develop a convective envelope, a convective core, or both. Low-mass stars are fully convective, giants may accommodate several distinct convective shells. In the Sun, the energy transport in the inner parts is entirely by radiation, while in the outer $30 \%$ (in radius) it is primarily carried by large-scale convective flows. At the surface, the solar granulation is the visible imprint of hot rising and cool sinking gas in the outermost layers of the convection zone.

The role of stellar convection is manifold: convective energy transport determines the internal structure of a star and in part its radius; convective regions are chemically completely mixed, and overshooting convective flows can lead to partial mixing of the adjacent radiative layers; convective motions and concomitant temperature inhomogeneities exert a direct influence on stellar spectra, causing small but practically relevant changes in wavelength, shape, and strength of spectral lines; stochastic convective motions excite stellar oscillations and are a source of acoustic energy, contributing to the heating of stellar chromospheres. In combination with differential rotation, convection is a necessary precondition for the operation of the magnetic dynamo, and hence for stellar activity.

Unfortunately, a rigorous theory of stellar convection is lacking due to the complexity of the underlying hydrodynamical problem. So far, stellar structure models still rely largely on a simplistic approach, the so called mixing-length theory (MLT), or variants thereof. Alternatively, radiation hydrodynamics (RHD) simulations based on first principles can now provide realistic, physically consistent ab initio models of stellar convection which can be applied to a variety of problems in stellar astrophysics. They can also serve to guide the development of more advanced convection theories, and to check their validity. 

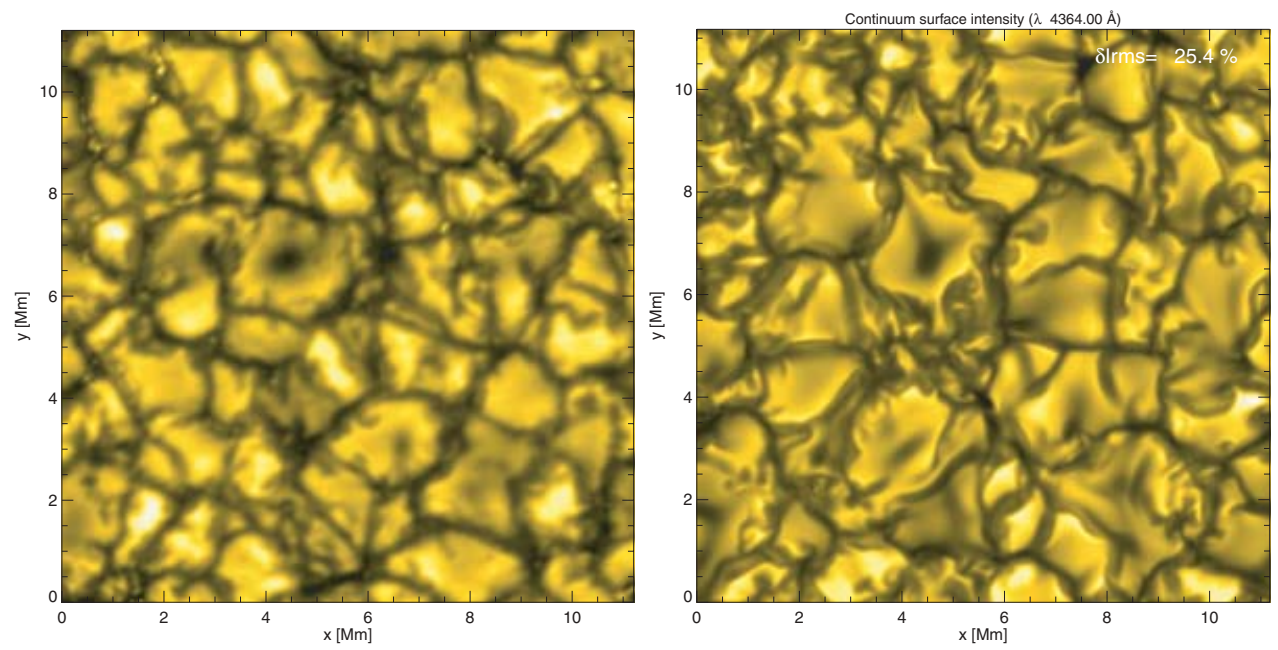

Figure 1. Left: Quiet solar granulation as observed with the $1 \mathrm{~m}$ Swedish Solar Telescope (courtesy Mats Carlsson 2004). Right: High-resolution $\mathrm{CO}^{5}$ BOLD simulation of solar surface convection on a $n_{\mathrm{x}} \times n_{\mathrm{y}} \times n_{\mathrm{z}}=400 \times 400 \times 165$ grid. Both images show the emergent continuum intensity (using identical scaling) at $\lambda 4364 \AA$ in a field measuring $15^{\prime \prime} \times 15^{\prime \prime}(11 \times 11 \mathrm{Mm})$.

\section{Numerical simulation of stellar surface convection with $\mathrm{CO}^{5} \mathrm{BOLD}$}

Stellar surface convection is governed by the conservation equations of hydrodynamics and radiative energy transfer. The radiation hydrodynamics code $\mathrm{CO}^{5} \mathrm{BOLD}$ is developed for realistic simulations of stellar convection (Freytag, Steffen \& Dorch 2002; Wedemeyer et al. 2004). Like 'classical' 1D model atmospheres, the resulting 3D ab initio models are characterized by $T_{\text {eff }}, \log g$, and chemical composition, but the need for additional free physical parameters like $\alpha_{\mathrm{MLT}}, \xi_{\text {micro }}$, and $\xi_{\text {macro }}$ is eliminated. The 3D simulations include all essential microphysics and are designed for a direct comparison with real stars.

Based on a Godunov-type finite volume approach, the code provides the time-dependent solution for a compressible radiating fluid in an external gravity field on a fixed, nonstaggered 3D Cartesian grid (allowing variable spacing). Operator splitting separates Eulerian hydrodynamics, optional tensor viscosity, and radiation transport. Directional splitting decomposes the 3D hydrodynamics problem into 1D sub-steps which are treated by an approximate Riemann solver of Roe type, modified to work with an arbitrary equation of state, and to properly handle an external gravity field. This scheme is very robust and well adapted to handle transonic flows and shocks in a highly stratified medium. By design, the code guarantees the numerical conservation of mass, momentum, and energy.

For any prescribed chemical composition, $\mathrm{CO}^{5} \mathrm{BOLD}$ uses a tabulated equation of state taking into account partial ionization of $\mathrm{HI}$, He I, and He II, as well as the formation and dissociation of $\mathrm{H}_{2}$ molecules. The release of ionization energy (latent heat) is an important factor that influences the onset and strength of stellar convection.

The role of radiation in the hydrodynamical simulations is to describe the energy balance due to radiative heating and cooling. Noting that simplifications like the diffusionor Eddington approximation are not valid in the critical layers around optical depth unity, the rate of radiative energy exchange, $\boldsymbol{\nabla} \cdot \boldsymbol{F}_{\text {rad }}$, is computed from the solution of the nonlocal transfer equation on a system of rays (typically using 6 polar and 4 azimuthal angles, resulting in $9 \times n_{\mathrm{x}} \times n_{\mathrm{y}}$ long rays). We use realistic stellar opacities, optionally based on ATLAS or MARCS opacity distribution functions. The frequency dependence of the radiation field is treated by a so called multi-group method, where frequencies are sorted 

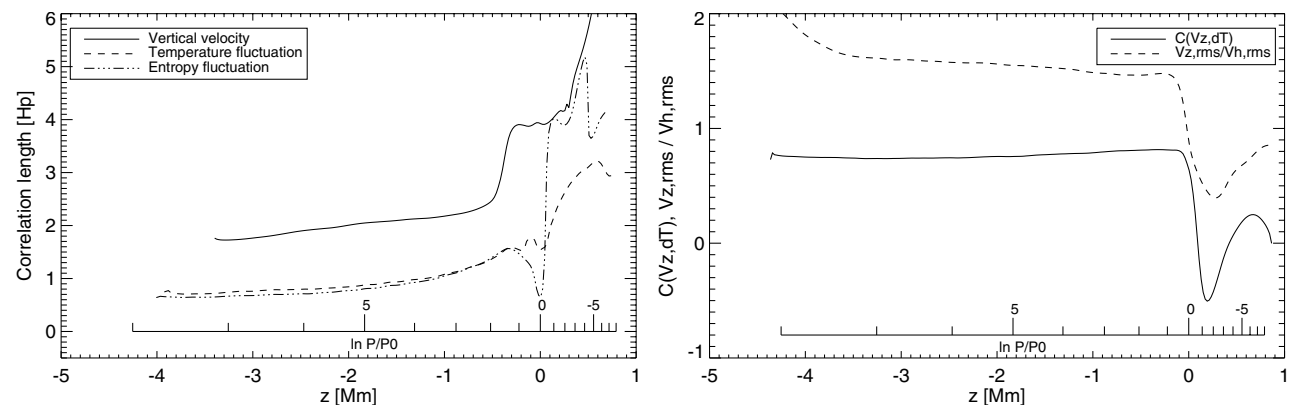

Figure 2. Left: Depth dependence of the FWHM (in units of the local pressure scale height $\left.H_{\mathrm{p}}\right)$ of the two-point spatial correlation functions $C\left(V_{\mathrm{z}}, V_{\mathrm{z}}\right)$ (solid), $C(\Delta T, \Delta T)$ (dashed), and $C(\Delta s, \Delta s)$ (dot-dashed), representing the vertical correlation length of vertical velocity, temperature and entropy fluctuations, respectively. Right: Local correlation coefficient $C\left(V_{\mathrm{z}}, \Delta T\right)$ of vertical velocity and temperature fluctuations (solid), and ratio of vertical to horizontal velocity, $V_{\mathrm{z}, \mathrm{rms}} / V_{\mathrm{h}, \mathrm{rms}}$ (dashed; $\left.V_{\mathrm{h}, \mathrm{rms}}^{2}=\left(V_{\mathrm{x}, \mathrm{rms}}^{2}+V_{\mathrm{y}, \mathrm{rms}}^{2}\right) / 2\right)$ as a function of geometrical height. Data derived from a $\mathrm{CO}^{5} \mathrm{BOLD}$ radiation hydrodynamics simulation of solar surface convection on a $200 \times 200 \times 250(11 \times 11 \times 5 \mathrm{Mm})$ grid.

into a small number of bins (typically $4 \ldots 6$ ) according to the ratio of monochromatic to Rosseland optical depth (opacity binning). So far, strict LTE is assumed, thus scattering cannot be treated. Radiation pressure is ignored. This method provides a simplified, yet realistic description of non-local, frequency-dependent radiative transfer in 3 dimensions.

The code is written in ForTRAn90 and is parallelized by OpenMP directives. Advanced features include a magnetohydrodynamics module and a chemical reaction network that allows the investigation of non-equilibrium molecule formation in a dynamic environment (for more details see http://www.astro.uu.se/ bf/co5bold_main.html).

The results shown in the following were obtained with $\mathrm{CO}^{5} \mathrm{BOLD}$ in the so-called 'boxin-a-star' setup, where the computational domain consists of a tiny Cartesian section of the star located near the surface. The lateral boundaries of the box are periodic, while upper and lower boundaries can be open (transmitting) or closed (reflecting), depending on the particular application. The high degree of realism achieved by today's simulations is illustrated in Fig. 1, showing a comparison of observed and synthetic solar granulation.

It is worth mentioning that $\mathrm{CO}^{5} \mathrm{BOLD}$ can also operate in the 'star-in-a-box' setup, offering the possibility to include an entire star in a Cartesian computational box in order to perform global convection simulations, as pioneered by B. Freytag for red supergiants (Freytag, Steffen \& Dorch 2002; Freytag 2006). Global simulations of this kind are computationally very challenging and still in a more experimental state than the local simulations that are discussed below.

\section{3D hydrodynamical simulations versus $1 \mathrm{D}$ mixing-length models}

Numerical simulations of stellar convection can serve to check the validity of the mixing-length theory, and to quantify the expected errors. Using idealized simulations of efficient convection, Chan \& Sofia (1987) found support for the assumption that the mixing length, $\ell$, scales with the local pressure scale height $H_{\mathrm{p}}$. Defining the correlation coefficient $C\left(q_{1}, q_{2}\right) \equiv\left\langle q_{1} q_{2}\right\rangle /\left(\sqrt{\left\langle q_{1}^{2}\right\rangle} \sqrt{\left\langle q_{2}^{2}\right\rangle}\right.$, where $\langle$.$\rangle denotes the combined average over$ time and horizontal position, they evaluated the two-point correlation $C\left(V_{\mathrm{z}, 1}, V_{\mathrm{z}, 2}\right)$, measuring the correlation between the vertical velocity at two different heights. They found that the velocity correlation length, defined as the full width at half maximum of this correlation function, is about $\ell_{\mathrm{V}} \approx 2 \mathrm{Hp}$. In a follow-up paper, Chan \& Sofia (1989) 

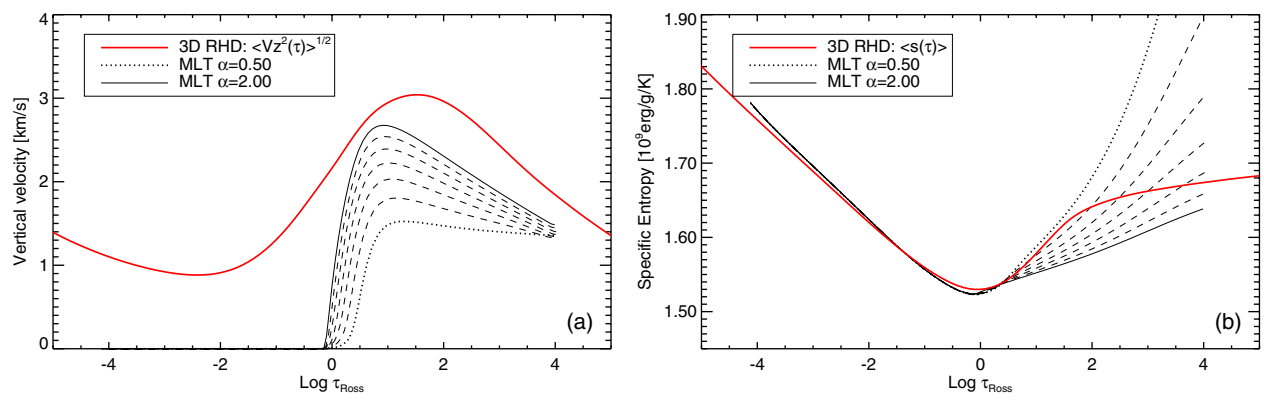

Figure 3. a: RMS vertical velocity derived from a $C \mathrm{O}^{5} \mathrm{BOLD}$ simulation of solar surface convection as a function of Rosseland optical depth (thick solid) compared with the convective velocity predicted by MLT (in the version by Mihalas 1978) for different values of the mixing-length parameter $\alpha_{\text {MLT }}$ between 0.5 (dotted) and 2.0 (thin solid) in steps of 0.25 . b: Similar $3 \mathrm{D} / 1 \mathrm{D}$ comparison of the entropy stratification, again evaluated on the $\tau_{\text {Ross }}$ scale.

found the corresponding correlation lengths of the temperature and entropy fluctuations to scale similarly, $\ell_{\Delta \mathrm{T}} \approx \ell_{\Delta \mathrm{s}} \approx 1.5 \mathrm{Hp}$. In Fig. 2 (left), we show the same correlation lengths derived from a realistic $\mathrm{CO}^{5} \mathrm{BOLD}$ simulation of the solar granulation. In the optically thick subsurface layers $(z<-0.5 \mathrm{Mm}), \ell$ decreases slightly with depth for all quantities. The correlation lengths $\ell_{\Delta \mathrm{T}}$ and $\ell_{\Delta \mathrm{s}}$ are very similar and by roughly a factor of 2 smaller than $\ell_{\mathrm{V}}$. The fact that $\ell_{\mathrm{V}}$ and $\ell_{\Delta \mathrm{T}}$ have significantly different values is incompatible with the 'bubble' concept of MLT. We confirm the finding by Chan \& Sofia $(1987,1989)$ that the local correlation coefficient between vertical velocity and temperature fluctuation is $C\left(V_{\mathrm{z}}, \Delta T\right) \approx 0.8$, which seems to be a very robust result (Fig. 2, right). The figure also shows that, contrary to naive expectations, vertical and horizontal velocities are of similar size even in the deep layers.

A differential comparison of the convective velocity predicted by MLT for various values of the mixing-length $\ell=\alpha_{\mathrm{MLT}} H_{\mathrm{p}}$ with the rms vertical velocity obtained from a 3D $\mathrm{CO}^{5} \mathrm{BOLD}$ simulation of solar surface convection is shown in Fig. 3a. Differential means that the MLT models were computed with exactly the same physics (EOS, opacities) and numerical methods (radiative transfer module and opacity binning scheme) as the 3D simulations, the only real difference being the treatment of convection (MLT vs. hydrodynamics). In the convectively stable layers $\left(\log \tau_{\text {Ross }}<0\right)$, the velocities vanish by definition in MLT, whereas substantial velocities are predicted by the hydrodynamical simulations owing to convective overshoot and waves. The latter are responsible for the increase of $V_{\text {rms }}$ above $\log \tau_{\text {Ross }} \approx-2$. In the convectively unstable layers $\left(\log \tau_{\text {Ross }}>0\right)$, the correspondence between 1D MLT and 3D RHD is clearly much better, albeit far from perfect. An optimum agreement would require $\alpha_{\mathrm{MLT}}>2$, but still a difference in the location of the velocity maximum of $\approx 70 \mathrm{~km}$ remains.

A corresponding comparison of the thermal structure is shown in Fig. 3b. In the subphotospheric layers, no choice of $\alpha_{\mathrm{MLT}}$ can reproduce the mean entropy profile of the RHD simulations. Figure $3 \mathrm{~b}$ suggests that the effective mixing-length increases strongly with depth, from $\alpha_{\mathrm{MLT}} \approx 0.5$ to 2.0 . This naive interpretation is, however, not supported by the depth-dependence of the correlations lengths presented in Fig. 2 (left).

In the stable layers, the mean entropy stratification obtained from the 3D hydrodynamical model is in close agreement with MLT predictions, indicating that the photosphere is essentially in radiative equilibrium despite substantial convective overshoot. However, the situation changes dramatically as the chemical composition of the stellar matter becomes metal-poor. This is demonstrated in Fig. 4: for the RHD simulation of the 'metalpoor sun', the mean photospheric temperature is up to $1000 \mathrm{~K}$ below the MLT result, 

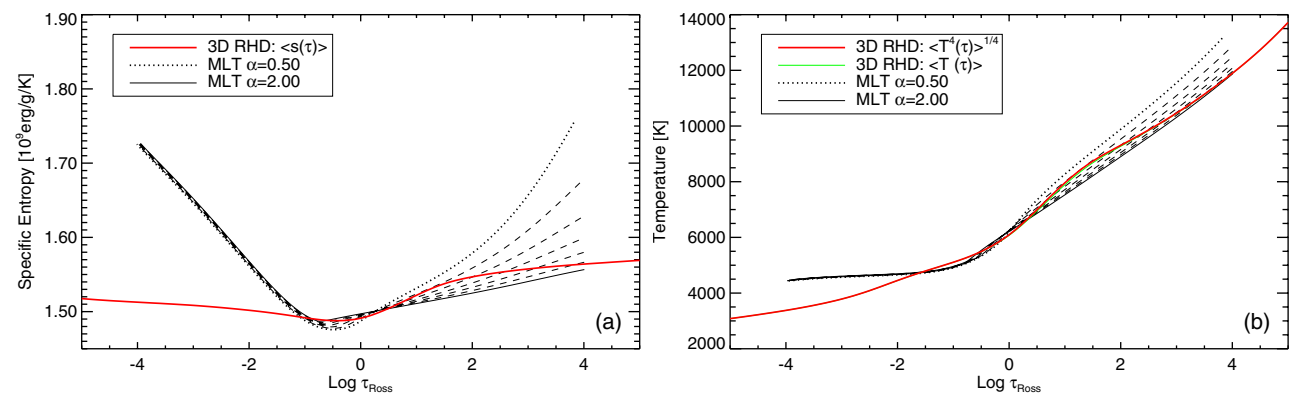

Figure 4. Mean thermal structure of the surface layers of a 'metal-poor sun' ( $T_{\text {eff }}=5770 \mathrm{~K}$, $\log g=4.44,[\mathrm{M} / \mathrm{H}]=-2$ ) as derived from a $\mathrm{CO}^{5} \mathrm{BOLD}$ convection simulation (thick/red) and from MLT models computed for different values of $\alpha_{\mathrm{MLT}}$ (thin/black). Both the mean entropy stratification (a) and the mean temperature structure (b) are evaluated on the $\tau_{\text {Ross }}$ scale.
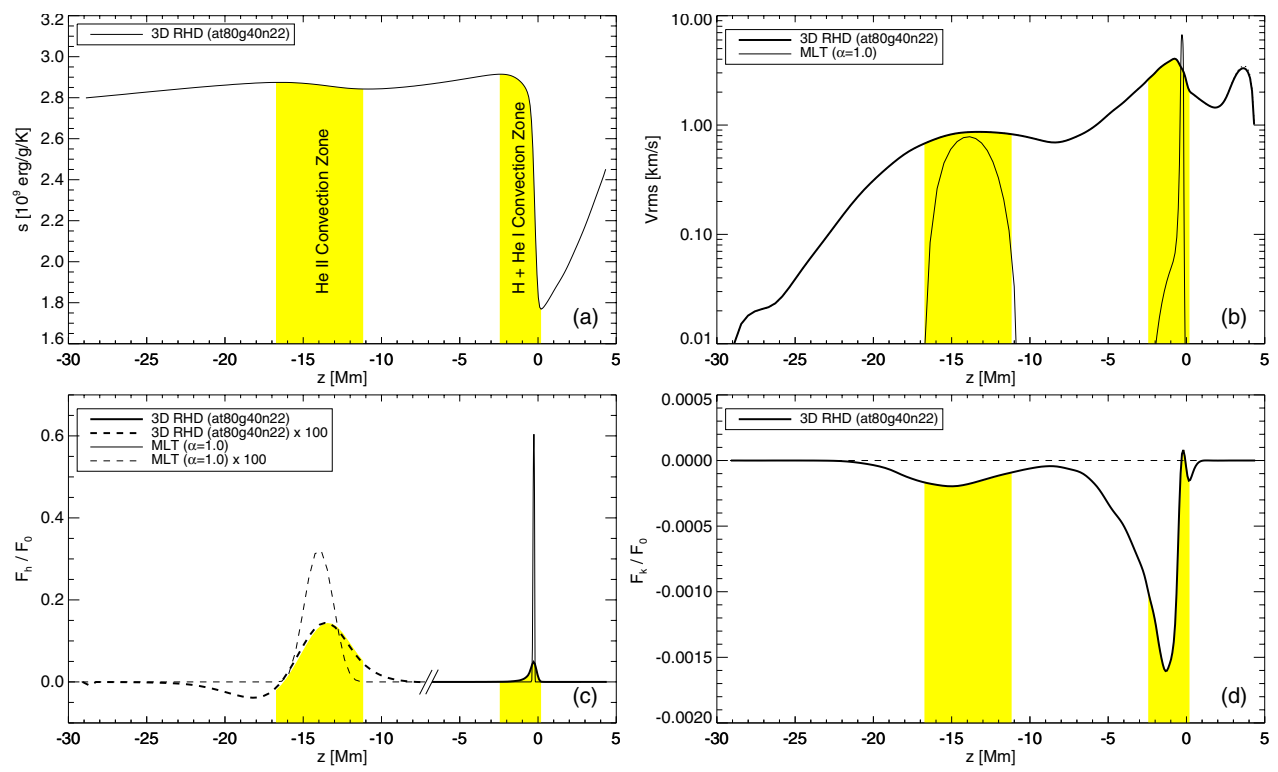

Figure 5. Depth profiles of different quantities derived from a $\mathrm{CO}^{5} \mathrm{BOLD}$ simulation of the convective surface layers of a main-sequence A-type $\operatorname{star}\left(T_{\text {eff }}=8000 \mathrm{~K}, \log g=4.00,[\mathrm{M} / \mathrm{H}]=0\right)$ by averaging over time and horizontal position. (a): specific entropy, defining the location of two separate convection zones (shaded regions) where $\mathrm{d} s / \mathrm{d} z<0 ;(\mathbf{b})$ : logarithmic vertical velocity; (c): enthalpy flux $\left\langle\rho V_{\mathrm{z}} h\right\rangle$ (multiplied by 100 below $z=-7 \mathrm{Mm}$ ); (d): flux of kinetic energy $\left\langle\rho V_{\mathrm{z}}^{3} / 2\right\rangle$. Thin lines in panels (b) and (c) show the MLT results for $\alpha_{\mathrm{MLT}}=1$ for comparison.

indicating severe deviations from radiative equilibrium. Obviously, the lack of sufficiently strong spectral lines renders the radiative heating too inefficient to compensate the expansion cooling due to overshoot, even though the convective velocities are somewhat smaller than for solar metallicity. Clearly, this drastic deviation from the 'classical' temperature stratification in the line forming layers has serious consequences for spectroscopic abundance determinations (see also Asplund et al. 1999).

Conditions are rather different in the surface layers of early main-sequence A-type stars, which are characterized by two distinct shallow convection zones, the upper one driven by H I + He I ionization, the lower one by He II ionization. In this case, both convection zones, together with sufficiently thick radiative layers above and below, can be included in a single model. Such a configuration is ideally suited to study overshoot and the 

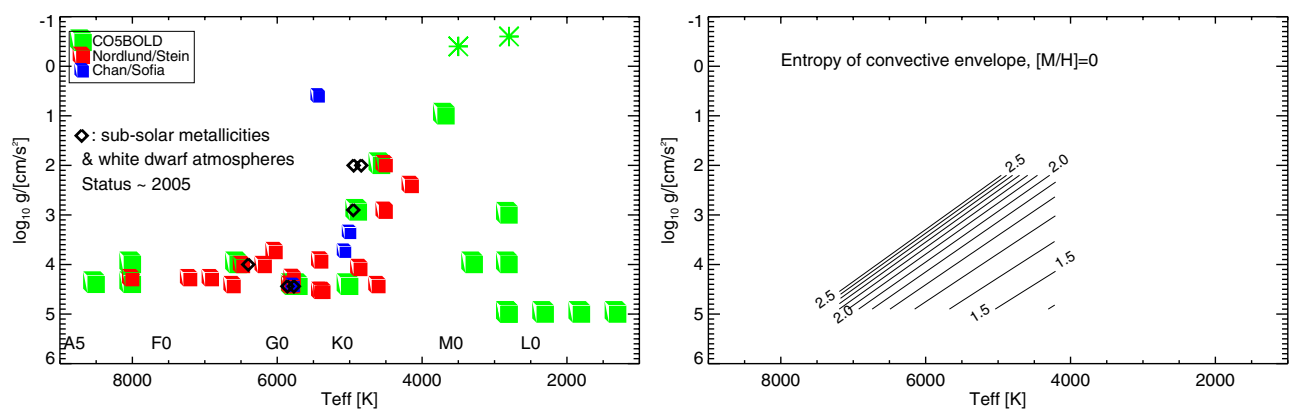

Figure 6. Left: Distribution in the Hertzsprung-Russell diagram of realistic 3D stellar surface convection models computed by different groups and codes (status $\approx 2005$ ). Right: Contours in the HRD of the entropy (in units of $10^{9} \mathrm{erg} / \mathrm{g} / \mathrm{K}$ ) of the convective envelope for stars with solar composition, as derived from 2D simulations by Ludwig, Freytag, \& Steffen (1999).

interaction of two convection zones separated by a radiative buffer layer. Since the lower layers are convectively stable, a closed lower boundary is sufficient. RHD simulations for A-type stars are time consuming because the radiative time scales are much shorter than the hydrodynamic time scales, $\tau_{\text {rad }} \ll \tau_{\text {hyd }}$, whereas $\tau_{\text {rad }} \approx \tau_{\text {hyd }}$ for solar type stars.

First 3D simulations for A-type stars have been presented by Steffen, Freytag, \& Ludwig (2005) and Freytag, \& Steffen (2005). In Fig. 5 we show preliminary results of the first A-star simulation employing frequency-dependent radiative transfer. We conclude, again, that the radiative layer between the two convection zones is completely mixed, and that overshoot is characterized by an exponential decay of the velocity amplitude with depth (Fig. 5b). Energetically, the convection zones are well separated. Convection is inefficient, contributing no more than $5 \%$ of the energy flux in the upper, and less than $0.2 \%$ in the lower convection zone (Fig. $5 \mathrm{c}$ ). The flux of kinetic energy is roughly 10 times smaller than the enthalpy flux, but negative throughout (Fig. 5d). The strictly differential comparison demonstrates that MLT fails miserably under these conditions, but also the more advanced convection theory by Kupka \& Montgomery (2002) gives incorrect fluxes (see Steffen, Freytag, \& Ludwig 2005). Synthetic line profiles based on the new A-star simulation are compared with observations by Kochukhov et al. (this volume).

\section{Applications of 3D simulations of stellar surface convection}

Realistic simulations of stellar surface convection can not only serve as a test bed for MLT and more advanced convection theories but can also be applied directly to a number of problems in stellar astrophysics. In the meantime, convection simulations are no longer restricted to the Sun but cover a substantial area of the Hertzsprung-Russell diagram (HRD) as illustrated in Fig. 6 (left). In the context of stellar evolution, hydrodynamical convection models can be employed to derive the adiabat of the convective stellar envelope, as shown by Ludwig, Freytag, \& Steffen (1999). The right panel of Fig. 6 shows the results obtained by these authors from $2 \mathrm{D}$ simulations of surface convection in solar metallicity stars. If desired, the entropy of the convection zone may be converted into an effective mixing-length parameter $\alpha_{\text {MLT }}^{*}$ to be used with standard evolution calculations based on MLT in order to recover the correct adiabat. In this sense, RHD simulations provide a theoretical prediction of how $\alpha_{\mathrm{MLT}}^{*}$ varies across the HRD. It would certainly be worthwhile to extend the study by Ludwig, Freytag, \& Steffen (1999), utilizing 3D simulations and covering a larger region of the HRD, in particular towards lower gravities.

RHD simulations have the advantage of providing information far beyond the scope of MLT. They have been employed to study the properties of overshoot and its 
mixing efficiency in A-type stars and White Dwarfs (e.g. Freytag, Ludwig \& Steffen 1996). They can also be used to investigate the variation of the stellar granulation pattern as a function of spectral type, including the size of convection cells at the surface and their evolution time scales. Recently, realistic convection simulations have been exploited to predict the micro-variability due to stellar granulation (Ludwig 2006). This information about the photometric 'noise' is of particular relevance in the context of current and future highly sensitive searches for extra-solar planets and stellar p-mode oscillations from space. Further applications include the prediction of the excitation rates of stellar p-modes (e.g. Samadi/Stein, this volume), and the simulation of wave propagation from the convection zone into the upper atmosphere in order to clarify their role for structure, dynamics, and heating of the solar chromosphere (Wedemeyer et al. 2004). Finally, 3D dynamic atmospheres have been successfully utilized for the interpretation of stellar interferometric data (e.g. Aufdenberg, Ludwig, \& Kervella 2005), and for accurate spectroscopic abundance determinations (see below).

\section{3D model atmospheres and the solar oxygen abundance}

The deficiencies of 'classical' 1D model atmospheres are a consequence of the crude MLT treatment of convection. As shown in Sect. 3, no choice of the free parameter $\alpha_{\mathrm{MLT}}$ can reproduce the correct mean temperature structure, let alone the correct velocity field in the line-forming regions, which instead needs to be represented in terms of microand macroturbulence by the fudge parameters $\xi_{\text {micro }}$ and $\xi_{\text {macro }}$. These problems can be overcome by using 'parameter-free' 3D radiation hydrodynamics simulations, which in addition take into account the effect of horizontal inhomogeneities implied by the stellar granulation pattern. Weak points of the 3D model atmospheres include the approximate treatment of radiative transfer (opacity binning) and the limited spatial resolution (unrealistic low Reynolds number).

Recent spectroscopic abundance determinations by Asplund et al. (2004), based on a $3 \mathrm{D}$ hydrodynamic model atmosphere, led to a much debated downward revision of the so$\operatorname{lar} \mathrm{C}, \mathrm{N}$, and $\mathrm{O}$ abundances. Their result for the oxygen abundance is $\log \epsilon_{\mathrm{O}}=8.66 \pm 0.05$ (on the scale $\log \epsilon_{\mathrm{H}}=12$ ), causing a dramatic deterioration of the agreement between the thermal structure derived from helioseismic inversions and theoretical solar models, respectively. Motivated by this problem, Caffau et al. (A\&A, in preparation) are currently using a 3D $\mathrm{CO}^{5} \mathrm{BOLD}$ simulation with 5-bin frequency-dependent radiative transfer based on MARCS opacities to see whether the results by Asplund et al. (2004) can be confirmed. This independent redetermination of the solar oxygen abundance is based on 2 forbidden and 7 permitted O I lines, using a number of different observations, including both disk-center ('intensity') and full-disk ('flux') spectra. In addition to 25 snapshots from the $\mathrm{CO}^{5} \mathrm{BOLD}$ simulation, we also derive abundances from different $1 \mathrm{D}$ atmospheres for comparison. Special care is taken to provide realistic error estimates.

The following preliminary conclusions can be drawn at this point: (i) 'intensity' and 'flux' spectra give practically the same result. (ii) the oxygen abundance derived from the $3 \mathrm{D} \mathrm{CO}^{5} \mathrm{BOLD}$ simulation is only slightly lower (by -0.04 dex) than that derived from the 1D empirical model by Holweger \& Müller (1974, HM), indicating that the 3D mean model and the 1D HM model have very similar temperature structures. (iii) the unknown cross sections for neutral particle collisions introduce uncertainties in the NLTE corrections for the O I triplet lines of up to 0.1 dex; depending on the weight of these lines, the resulting error in the mean oxygen abundance derived from our set of lines is about 0.05 dex. (iv) Our preliminary best estimate for the solar oxygen abundance is $\log \epsilon_{\mathrm{O}}=\mathbf{8 . 7 2} \pm \mathbf{0 . 0 6}$, which is close to the value recommended by Holweger (2001), 
$\log \epsilon_{\mathrm{O}}=8.736 \pm 0.078$. A remaining problem of our analysis is that the two forbidden [O I] lines give significantly different abundances, which cannot be explained by NLTE-effects or deficiencies of the model atmosphere. We hope to resolve this problem by analyzing the observed center-to-limb variations of these two line profiles.

\section{Acknowledgements}

Thanks to H.-G. Ludwig for providing the 1D MLT models for differential comparison, and to B. Freytag for the data of the latest A-Star simulation. The deep solar simulation was carried out at the CINECA Supercomputing Center in Bologna (Italy) by T. Straus.

\section{References}

Asplund, M., Grevesse, N., Sauval, A.J., Allende Prieto, C., \& Kiselman, D. 2004, A $\mathscr{E} A$ 417, 751 Asplund, M., Nordlund, A., Trampedach, R., \& Stein, R.F. 1999, A $3 A$ 346, L17

Aufdenberg, J.P., Ludwig, H.-G., \& Kervella, P. 2005, ApJ 633, 424

Chan, K., \& Sofia, S. 1987, Science 235, 465

Chan, K., \& Sofia, S. 1989, ApJ 336, 1022

Freytag, B., Ludwig, H.-G., \& Steffen, M. 1996, A\& A 313, 497

Freytag, B., Steffen, M. \& Dorch, B. 2002, Astron. Nachr. 323, 213

Freytag, B., \& Steffen, M. 2005, in: J. Zverko, J. Ziznovsky, S.J. Adelman \& W.W. Weiss (eds.), The A-star puzzle, Proc. IAU Symposium No. 224 (Cambridge University Press), p. 139

Freytag, B. 2006, A\&SA (in press)

Holweger, H., \& Müller, E. 1974, Solar Phys. 39, 19

Holweger, H. 2001 in: R.F. Wimmer-Schweingruber (ed.), Solar and galactic composition, AIP Conf. Proc. 598, p. 23

Kupka, F., \& Montgomery, M.H. 2002, MNRAS 330, L6

Mihalas, D. 1978, Stellar Atmospheres, 2nd edition (Freeman and Company)

Ludwig, H.-G. 2006, A\&A 445, 661

Ludwig, H.-G., Freytag, B., \& Steffen, M. 1999, A\&A $A 346,111$

Steffen, M., Freytag, B., \& Ludwig, H.-G. 2005, in: F. Favata, G.A.J. Hussain, \& B. Battrick (eds.), Proc. 13th Cambridge Workshop on Cool Stars, Stellar Systems and the Sun, (ESA Publications), p. 985

Wedemeyer, S., Freytag, B., Steffen, M., Ludwig, H.-G., \& Holweger, H. 2002, A\&A 414, 1121

\section{Discussion}

ROXBURGH: What exactly do you mean by an open bottom boundary condition ?

STEFFEn: The open bottom boundary allows advection into and out of the computational box. The net mass flux through the bottom is zero at all times. Gas entering the box has a fixed specific entropy; its value defines the effective temperature. Horizontal pressure fluctuations are suppressed. For the horizontal velocities we set $\partial u_{\mathrm{x}} / \partial z=\partial u_{\mathrm{y}} / \partial z=0$.

AsPLUND: I'm very pleased that you now with $\mathrm{CO}^{5}$ BOLD get the same result for the solar $\mathrm{O}$ abundance and metal poor stars as we got more than five years ago with the Nordlund-Stein code. A question: you didn't show a comparison between predicted and observed Fe lines - how good is the agreement for the line profiles and asymmetries?

STEFfEn: (i) Our oxygen abundance seems a bit higher than yours, probably owing to a slightly different mean temperature structure in the $\mathrm{CO}^{5}$ BOLD model. But you are right, our results agree within the error bars. (ii) We have computed synthetic line profiles of FeI $\lambda$ 5234, 5525, 6082, $6219 \AA$ with $\mathrm{CO}^{5}$ BOLD/Linfor3D and compared them to your results from the Nordlund-Stein code: the profiles agree almost perfectly, indicating very similar non-thermal line broadening. The line bisectors show only subtle differences. 